J. Klin. Endokrinol. Stoffw. 2018 $11: 94-100$ https://doi.org/10.1007/s41969-018-0036-5

(c) Der/die Autor(en) 2018, korrigierte Publikation 2018

CrossMark

Georg Zettinig ${ }^{1} \cdot$ Wolfgang Buchinger ${ }^{2} \cdot$ Michael Krebs $^{3}$

'Schilddrüsenpraxis Josefstadt, Wien, Österreich

${ }^{2} S$ childdrüseninstitut Gleisdorf, Gleisdorf, Österreich

${ }^{3}$ Klinische Abteilung für Endokrinologie und Stoffwechsel, Medizinische Universität Wien, Wien, Österreich

\title{
So wird die Feinnadelpunktion der Schilddrüse in Österreich durchgeführt - die Ergebnisse einer landesweiten Umfrage
}

allgemein gehaltene Empfehlungen, wie eine Feinnadelpunktion durchgeführt werden kann, aber keine einheitliche Leitlinie.

2011 wurde vom Robert Koch-Institut eine Empfehlung für Anforderungen an die Hygiene bei Punktionen und Injektionen veröffentlicht [7]. Aus den Formulierungen dieser Empfehlungen könnte abgeleitet werden, dass die ultraschallgezielte Feinnadelpunktion der Schilddrüse aus hygienischer Sicht als Organpunktion klassifiziert wird, was entsprechende hygienische Anforderungen wie bei Stanzbiopsien innerer Organe erfordern würde. Dies steht im Gegensatz zu verschiedenen Empfehlungen zur Durchführung von FNPs der Schilddrüse, wie den Anleitungen der American Thyroid Association [8]. Auch in Korea - jenem Land, in dem weltweit die meisten FNPs der Schilddrüse durchgeführt werden - wird im Konsensus-Statement der Korean Society of Thyroid Radiology die FNP hinsichtlich ihrer hygienischen Anforderungen wie eine intramuskuläre Injektion gestellt [9].

Die Österreichische Schilddrüsengesellschaft führte daher in Zusammenarbeit mit den anderen österreichischen Fachgesellschaften eine landesweite Umfrage durch, wie die FNP der Schilddrüse derzeit durchgeführt wird, um einen aktuellen Status zu erheben. wurde die Feinnadelpunktion in der modernen Thyreologie (wieder) etabliert. Die Feinnadelpunktion ist heute eine Basisuntersuchung bei der Abklärung fokaler Schilddrüsenläsionen und wird auch therapeutisch bei Zysten verwendet $[5,6]$.

Bezüglich der technischen Durchführung einer FNP der Schilddrüse werden zahlreiche verschiedene Varianten beschrieben, und die FNP der Schilddrüse wird sehr unterschiedlich durchgeführt. Die Dicke der Nadeln, die Volumina der Spritzen, die Anzahl der Punktionen pro Sitzung, die Zugangswege und auch die Hygiene werden von verschiedenen Punkteuren völlig unterschiedlich gehandhabt. Trotzdem führen diese verschiedenen Zugänge individuell $\mathrm{zu}$ sehr guter diagnostischer Aussage. Es gibt verschiedene, meist sehr

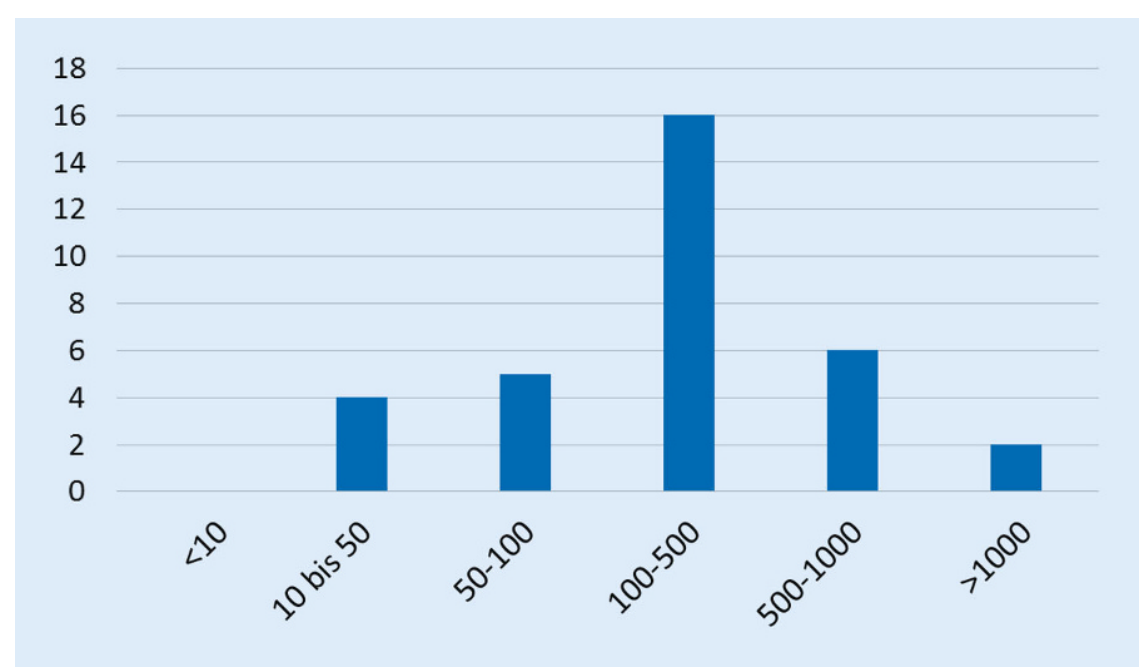

Abb. 1 ॥ Anzahl der Feinnadelpunktionen der Schilddrüse pro Jahr an den befragten Institutionen 


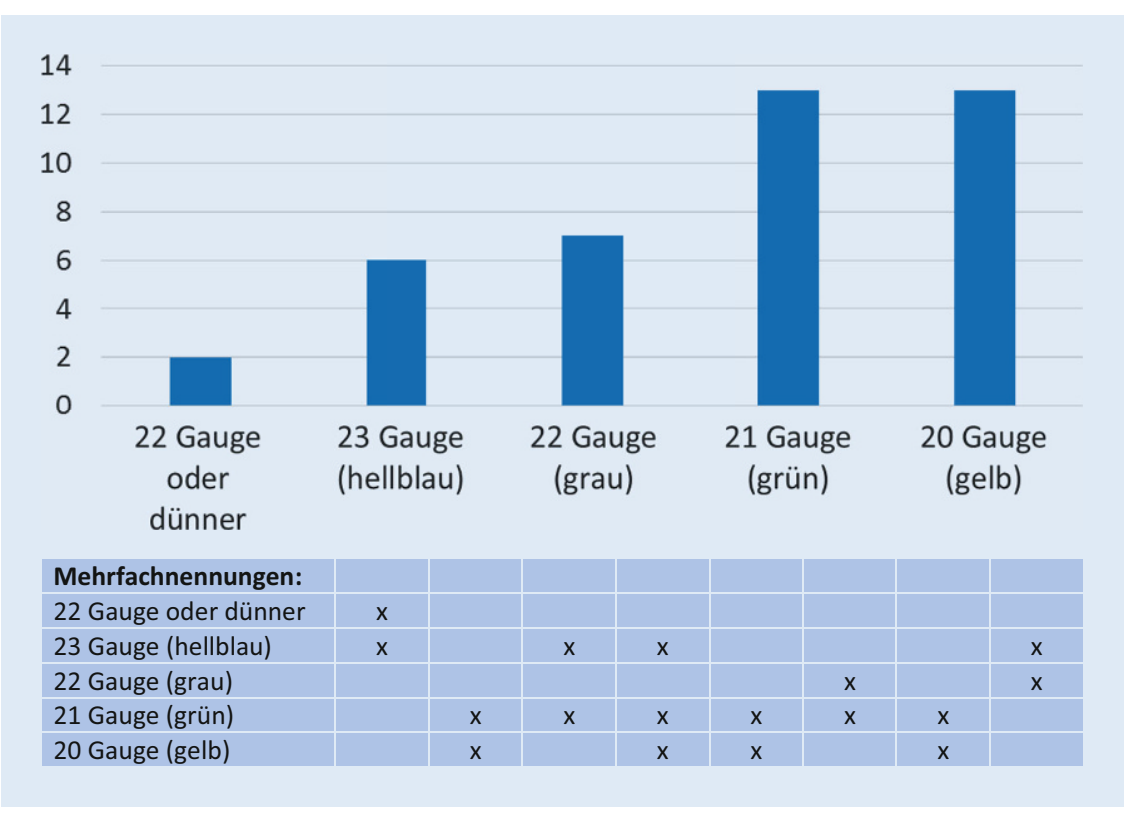

Abb. 2 \ Verwendete Nadeldicke in Gauge

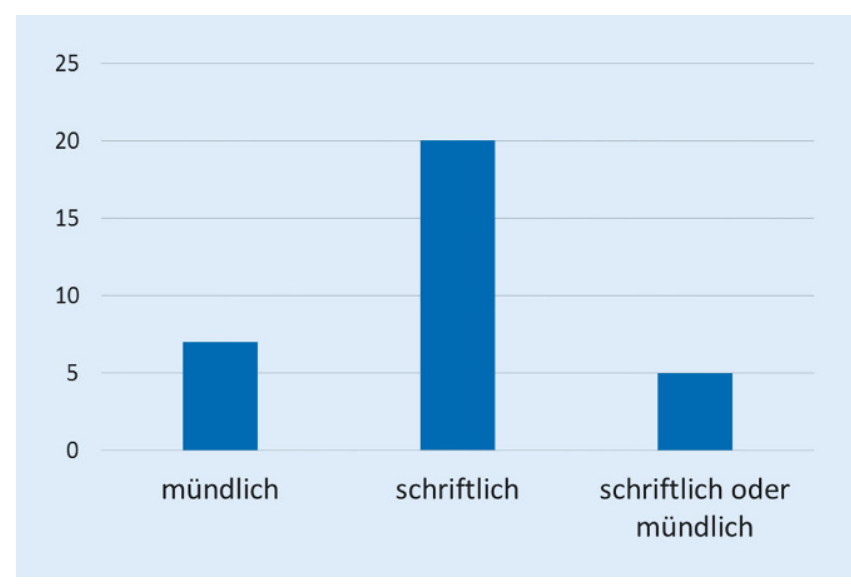

Abb. $3 \Delta$ Einverständnis der Patienten

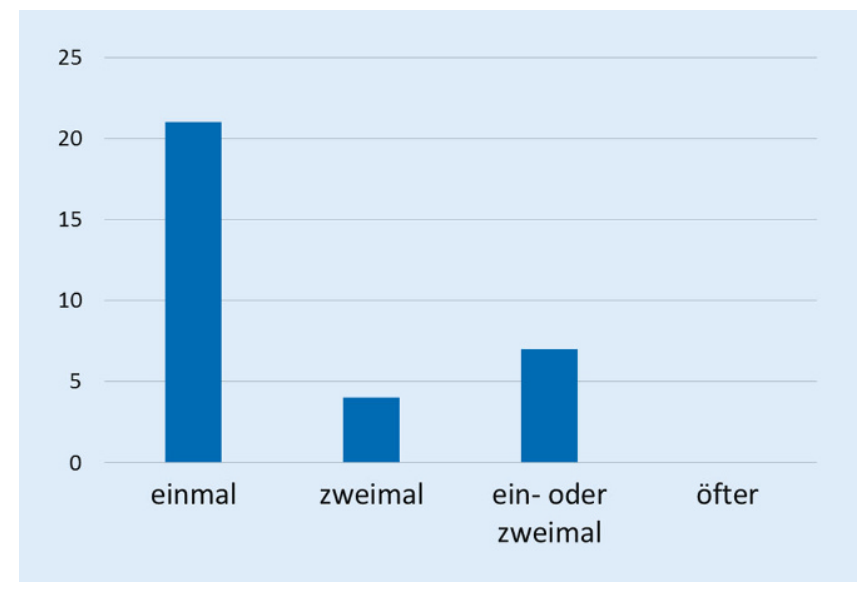

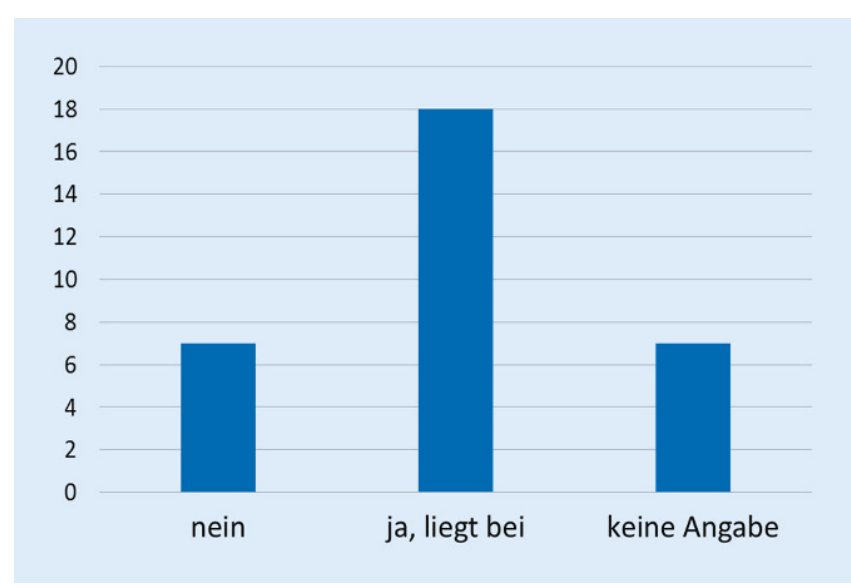

Abb. $4 \Delta$ Bereitstellung der verwendeten Einverständniserklärung für die Befragung

Abb. $5 \triangleleft$ Anzahl

der Punktionen pro

Knoten pro Sitzung 


\section{Aktuelles}

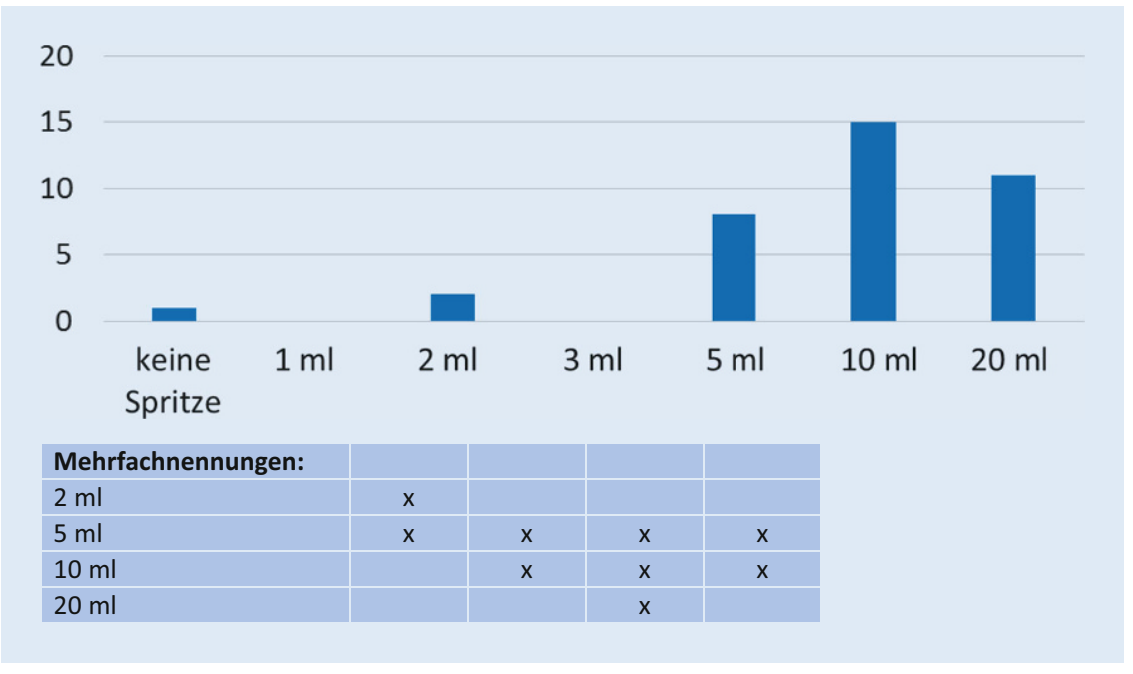

Abb. $6 \Delta$ Volumen der Spritze zur Aspiration bei Punktion solider Knoten
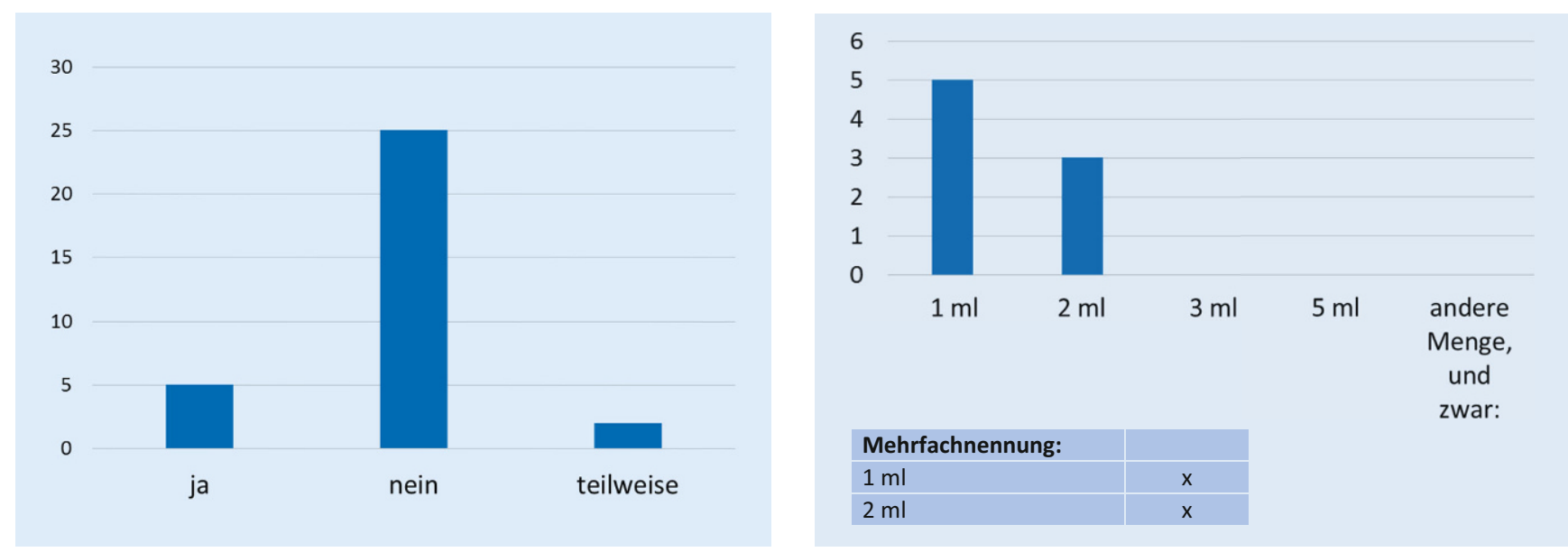

Abb. $7 \Delta$ Vorlegen von Luft in der Spritze vor dem Punktieren

Abb. 8 A Falls ja: Vorlegen von Luft in der Spritze in ml vor dem Punktieren

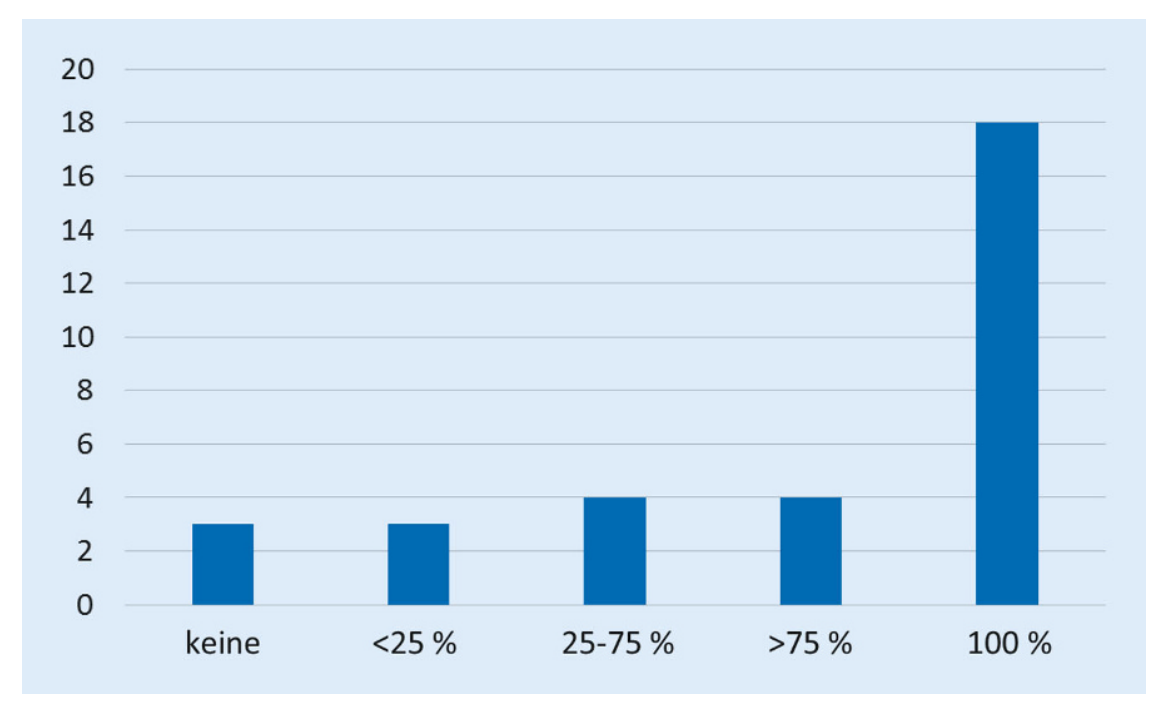

Abb. $9 \Delta$ Anteil der ultraschallgezielten Punktionen in Prozent 


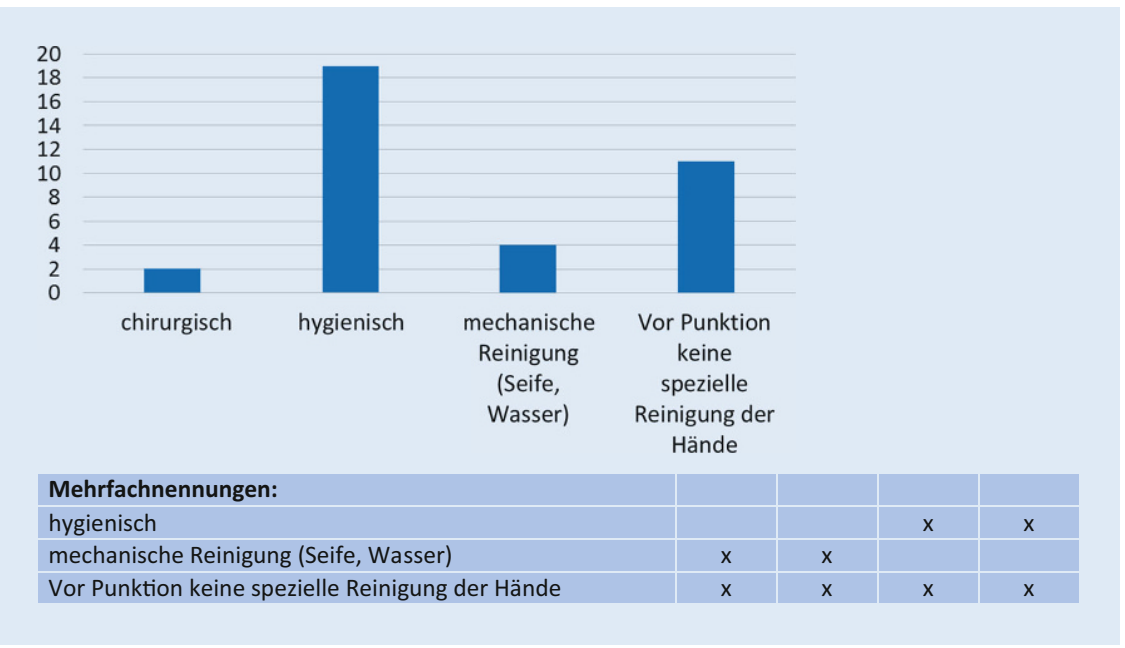

Abb. 10 ^ Händedesinfektion vor Punktion

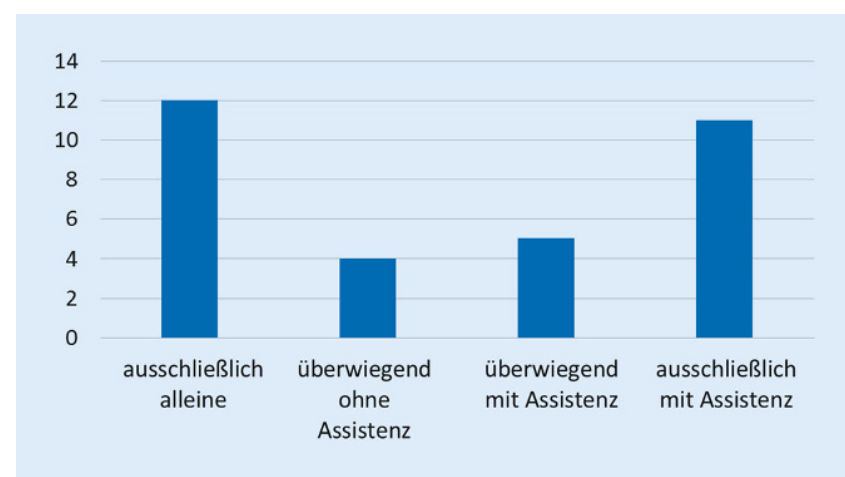

Abb. 11 ^ Punktion mit oder ohne Assistenz

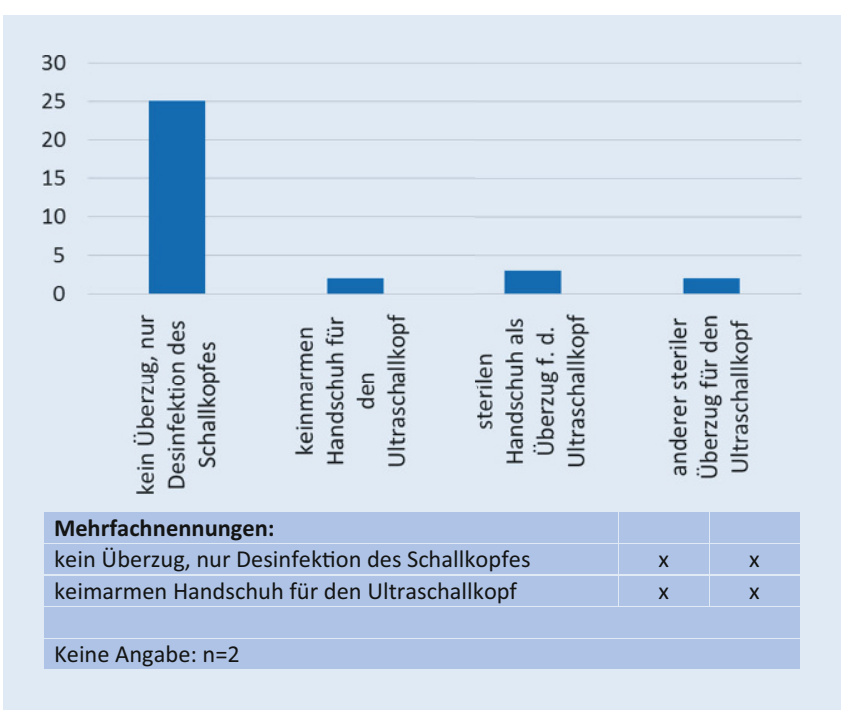

Abb. $13 \Delta$ Bei der Punktion verwendete Utensilien

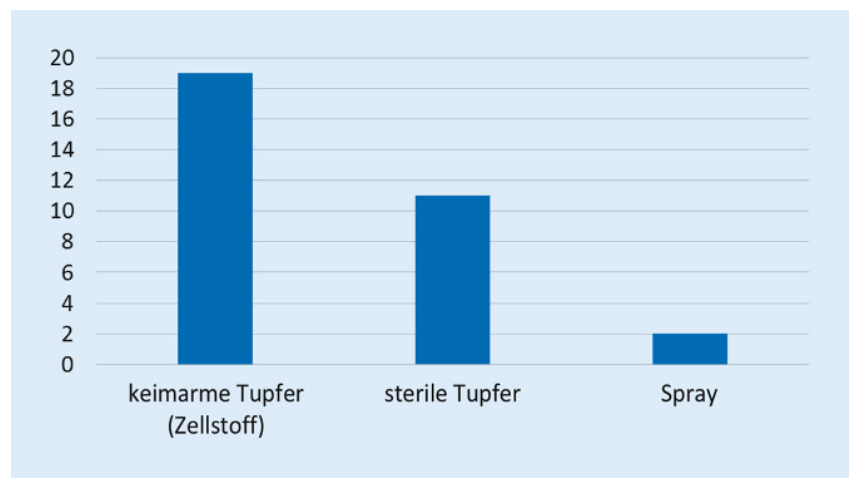

Abb. 12 ॥ Bei der Punktion verwendete Utensilien

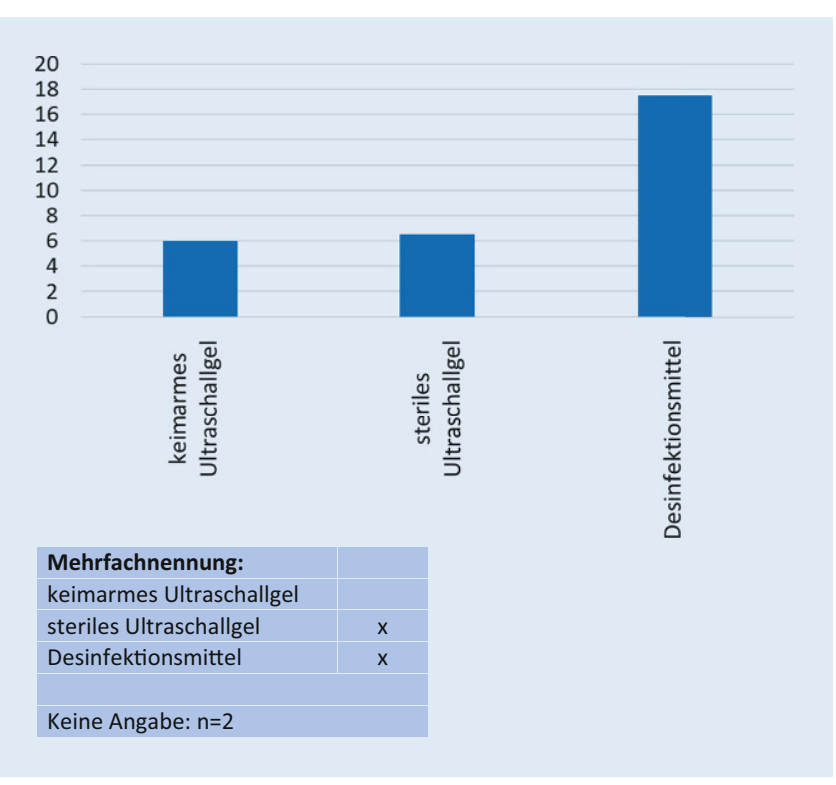

Abb. 14 A Bei der Punktion verwendete Utensilien 


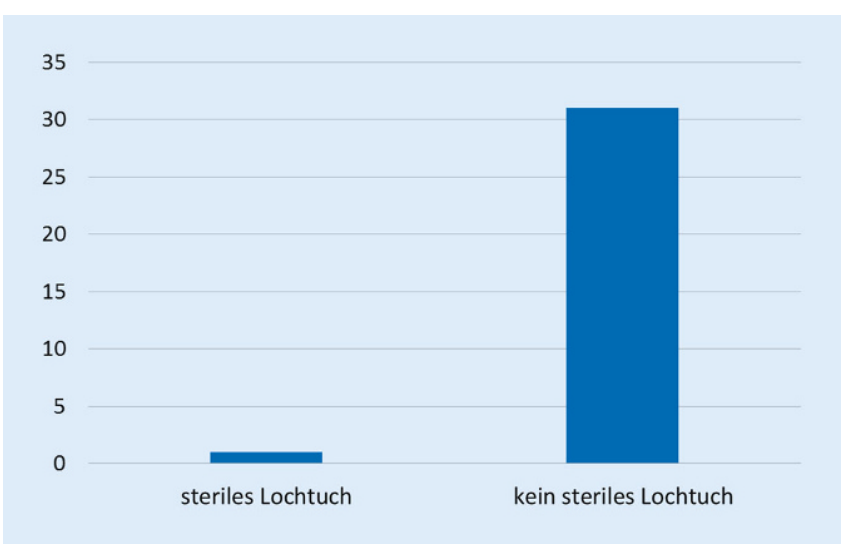

Abb. $15 \triangleleft$ Bei der Punktion verwendete Utensilien

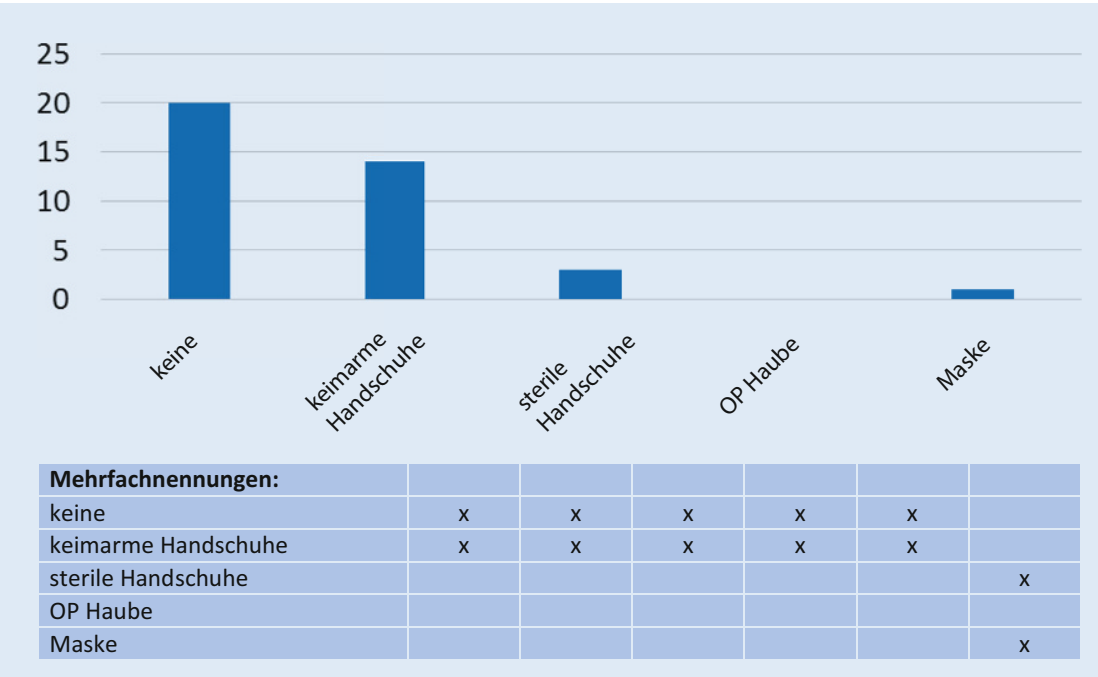

Abb. $16 \triangle$ Bei der Punktion verwendete Utensilien

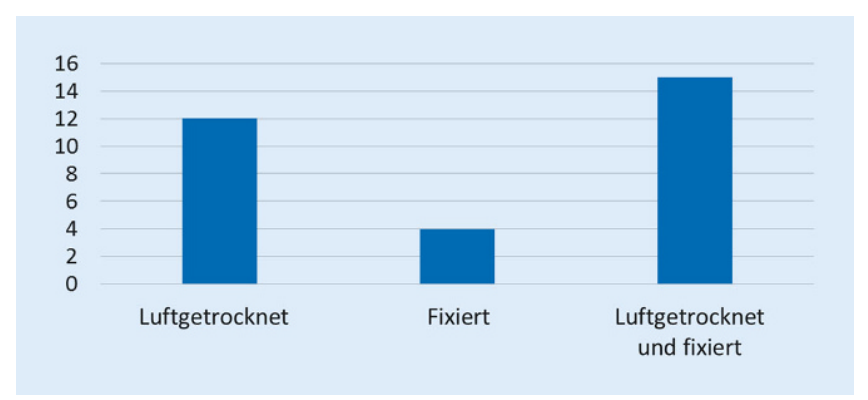

Abb. $17 \triangleleft$ Art der angefertigten Ausstriche

\section{Methoden}

In Absprache mit der Österreichischen Schilddrüsengesellschaft (OSDG), der Österreichischen Gesellschaft für Endokrinologie und Stoffwechsel (ÖGES), der Österreichischen Gesellschaft für Nuklearmedizin und Molekulare Bildgebung (OGNMB) und der Österreichischen Gesellschaft für Chirurgische Onkologie (ACO ASSO) erfolgte eine Aussendung

\section{Ergebnisse}

Die Ergebnisse dieser Umfrage sind in - Abb. 1, 2, 3, 4, 5, 6, 7, 8, 9, 10, 11, 12, 13, 14, $15,16,17,18,19$ und 20 dargestellt.

\section{Diskussion}

An der vorliegenden Umfrage haben alle österreichischen Institutionen teilgenommen, die FNPs der Schilddrüse in relevanter Zahl durchführen. Die Daten geben daher einen guten Überblick, wie die FNP in Österreich durchgeführt wird. Wie auch in anderen Ländern werden die verschiedensten Techniken angewandt, die den methodischen Empfehlungen der verschiedenen Fachgesellschaften entsprechen $[8,9]$.

Wie häufig FNPs bei thyreologischen Patienten in Österreich durchgeführt werden, schwankt zwischen den einzelnen Institutionen erheblich. Bei der Diskussion für zukünftige Leitlinien und Empfehlungen sollte dieser Umstand soweit möglich berücksichtigt werden. Die American Thyroid Association bezeichnete in den 2009 herausgegeben Leitlinien die FNP als die genaueste und kosteneffektivste Methode zur Abklärung von Schilddrüsenknoten [10]. In der aktuellen, im Jahre 2016 herausgegebenen Version der Leitlinien der amerikanischen Schilddrüsengesellschaft zur Knotenabklärung wird die FNP nicht mehr generell, sondern nach entsprechendem Sonografiebefund empfohlen [6].

Im Jahr 2016 wurde eine europaweite Umfrage der European Society of Radiology veröffentlicht, die unter ihren Mitgliedern die Infektionsprävention bei Ultraschalluntersuchungen erhob [11]. Auch hier zeigten sich große Unterschiede bei den Rückmeldungen. Bei Ultraschalluntersuchungen auf intakter Haut desinfizieren $29 \%$ der Rückmelder die Sonde erst am Ende des Tages. Bei endokavitärem Ultraschall desinfizieren $11 \%$, bei ultraschallbasierten Interventionen $6 \%$ der Einsender die Sonde nicht nach jedem Patienten. Ein Überzug des Schallkopfes wird nur bei $89 \%$ der endokavitären und nur bei $77 \%$ der interventionellen Untersuchungen verwendet. Bei diesen abgefragten interventionellen 


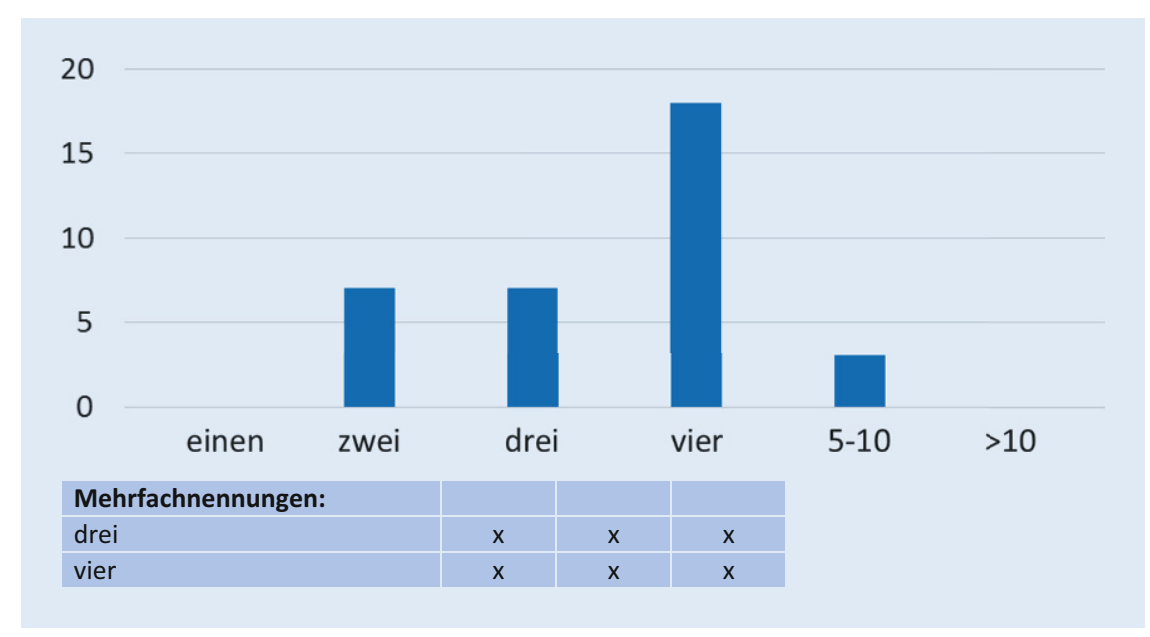

Abb. $18 \triangle$ Anzahl der angefertigten Ausstriche

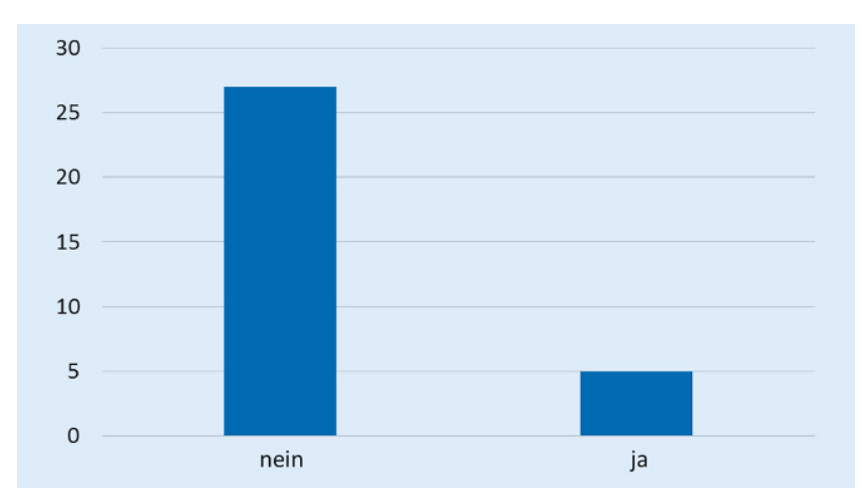

Abb. 19 \ Färbung und Beurteilung der Ausstriche vor Ort

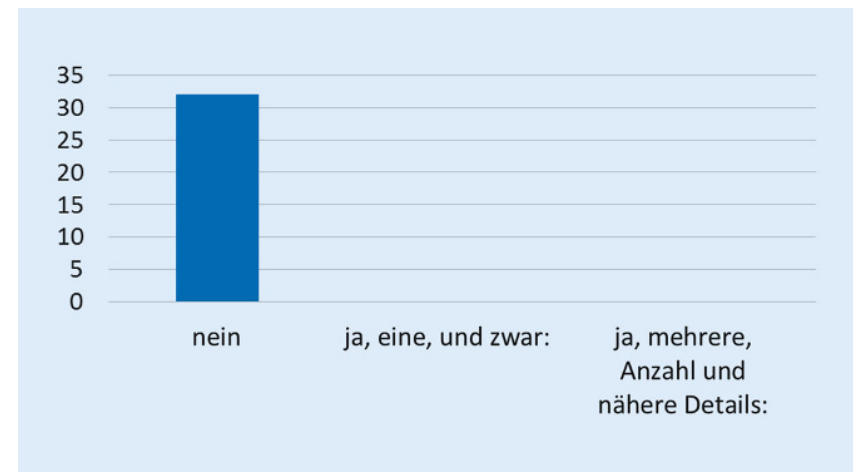

Abb. 20 \& Infektiöse Komplikation nach Punktion der Schilddrüse

\begin{abstract}
Expertenkommentar
von Prof. Dr. Markus Luster, Universitätsklinikum Marburg, Deutschland

Die Feinnadelpunktion der Schilddrüse ist integraler Bestandteil der Diagnostik von Schilddrüsenläsionen. In der Hand eines erfahrenen Untersuchers stellt sie ein effektives und wenig belastendes Verfahren zur Risikostratifizierung nodulärer Veränderungen des Organs dar. Im deutschsprachigen Raum wird sie tendenziell zu selten eingesetzt, insbesondere im Vergleich zu den sehr viel häufigeren chirurgischen Eingriffen an der Schilddrüse.

Den Autoren der österreichischen Erhebung gebührt Anerkennung für ihr Unternehmen, den Einsatz sowie die Rahmenbedingungen der Untersuchung näher zu beleuchten und Limitationen aufzuzeigen. Zu Recht wird darauf hingewiesen, dass spezifische Empfehlungen zur Feinnadelpunktion der Schilddrüse selten und zum Teil uneinheitlich sind und Handlungsanweisungen oftmals aus dem Kontext anderer Organpunktionen übertragen werden. Dies wird der klinischen Situation einer Schilddrüsenbiopsie nur selten gerecht.

Hinsichtlich der Indikationen zur Schilddrüsenpunktion darf auf eine kürzlich im Deutschen Ärzteblatt erschienene Arbeit von Feldkamp und Kollegen [16] hingewiesen werden: Als generelle Empfehlung kann gesagt werden, dass Schilddrüsenknoten unter $1 \mathrm{~cm}$ nur in Ausnahmefällen punktiert werden sollten. Zudem bleibt darauf hinzuweisen, dass in lodmangelgebieten (z.B. Deutschland) die Schilddrüsenszintigrafie mittels Technetium-99m-Pertechnetat als weichenstellende Untersuchung im Rahmen der Indikationsstellung gelten kann. Hyperfunktionelle Areale sollten wegen des sehr geringen Malignitätsrisikos und der häufig resultierenden zytologischen Diagnose "follikuläre Proliferation“ bzw. „Neoplasie“ nicht punktiert werden.

Der gezielte Einsatz der diagnostischen Verfahren Ultraschall, Szintigrafie und Feinnadelpunktion sollte langfristig zu einem Rückgang (unnötiger) chirurgischer Eingriffe führen [23], besonders wenn die Feinnadelpunktion unter standardisierten Rahmenbedingungen erfolgt.
\end{abstract}

Untersuchungen handelt es sich allerdings nicht um FNPs, sondern um andere interventionelle Ultraschalltechniken wie transvaginale Follikelpunktionen, transbronchiale Punktionen oder transrektale Prostatapunktionen. Bei diesen Techniken wurde im Gegensatz zur FNP der Schilddrüse auch die Transmission von Keimen berichtet 12-20].

Größtenteils werden die FNPs ultraschallgezielt durchgeführt, die hygienischen Rahmenbedingungen entsprechen denen einer intramuskulären Injektion und nicht einer Stanzbiopsie eines intraperitoneal gelegenen Organs, was sich ebenfalls mit den Empfehlungen der entsprechenden Fachgesellschaften deckt $[8,9]$. Allerdings gibt es unserer Meinung nach tatsächlich einzelne Defizite bezüglich allgemeiner hygienischer Aspekte. Die Leitlinien interventioneller Ultraschall der European Federation of Societies for Ultrasound in Medicine and Biology (EFSUMB) behandeln die 
FNP der Schilddrüse nicht, stellen aber im Teil I ihrer Leitlinien interventioneller Ultraschall einzelne grundlegende hygienische Forderungen [21]. Auch die Deutsche Gesellschaft für Ultraschall in der Medizin (DEGUM) veröffentlichte kürzlich Empfehlungen zur Hygiene in Sonografie und Endosonografie [22]. Diesen beiden und den Forderungen der European Society of Radiology schließen sich die Autoren an:

Adäquate Händehygiene ist essenziell. Es sollten ein steriler Überzug des Schallkopfes und steriles Ultraschallgel verwendet werden. Nach jeder FNP muss der Schallkopf desinfiziert werden.

\section{Schlussfolgerungen}

Die Feinnadelpunktion wird in Österreich auf unterschiedliche Art und Weise durchgeführt, und die Methodik entspricht den verschiedenen Empfehlungen der Fachgesellschaften [6, 8, 9]. Unsere Ergebnisse weisen darauf hin, dass auch in Österreich die FNP der Schilddrüse nicht wie eine Stanzbiopsie eines intraperitoneal gelegenen Organs klassifiziert wird, sondern mit einer intramuskulären Injektion vergleichbar ist. Die grundlegenden hygienischen Anforderungen für interventionelle Ultraschalltechniken müssen allerdings eingehalten werden.

\section{Korrespondenzadresse}

Univ.-Doz. Dr. Georg Zettinig

Schilddrüsenpraxis Josefstadt

Laudongasse 12/8, 1080 Wien, Österreich

kontakt@schilddruesenpraxis.at

\section{Einhaltung ethischer Richtlinien}

Interessenkonflikt. G. Zettinig, W. Buchinger und $M$. Krebs geben an, dass kein Interessenkonflikt besteht.

Dieser Beitrag beinhaltet keine von den Autoren durchgeführten Studien an Menschen oder Tieren.

Open Access. Dieser Artikel wird unter der Creative Commons Namensnennung 4.0 International Lizenz (http://creativecommons.org/licenses/by/4.0/deed. de) veröffentlicht, welche die Nutzung, Vervielfältigung, Bearbeitung, Verbreitung und Wiedergabe in jeglichem Medium und Format erlaubt, sofern Sie den/die ursprünglichen Autor(en) und die Quelle ordnungsgemäßnennen, einen Link zur Creative Com- mons Lizenz beifügen und angeben, ob Änderungen vorgenommen wurden.

\section{Literatur}

1. Diamantis A, Magiorkinis E, Koutselini H (2009) Fine-needle aspiration (FNA) biopsy: historical aspects. Folia Histochem Cytobiol 47(2):191-197

2. Kaadan AN (2004) Albucasis and thyroid surgery. 39th International Congress on the History of Medicine, Bari, Metaponto Italy (Paper)

3. Marketos S, Eftychiadis A, Koutras DA (1990) Thyroid diseases in the Byzantine era. J R Soc Med 83:111-113

4. Pauli Aegineta. Venetils, Libri Septem. Aldus 1528; Liber VI, f86

5. Russ G, Bonnema SJ, Erdogan MF, Durante C Ngu R, Leenhardt L (2017) European thyroid association guidelines for ultrasound malignancy risk stratification of thyroid nodules in adults: the EU-TIRADS. EurThyroid J 6(5):225-237. https://doi. org/10.1159/000478927

6. Haugen BR, Alexander EK, Bible KC, Doherty GM, Mandel SJ, Nikiforov YE, Pacini F, Randolph GW, Sawka AM, Schlumberger M, Schuff KG, Sherman SI, Sosa JA, Steward DL, Tuttle RM, Wartofsky L (2015) American thyroid association management guidelines for adult patients with thyroid nodules and differentiated thyroid cancer: The American thyroid association guidelines task force on thyroid nodules and differentiated thyroid cancer. Thyroid 26:1-133

7. Robert Koch-Institut (2011) Anforderungen an die Hygiene bei Punktionen und Injektionen. Empfehlung der Kommission für Krankenhaushygiene und Infektions-prävention beim Robert Koch-Institut (RKI). Bundesgesundheitsbl 54:1135-1144

8. Bernet V, Stancampiano F (2014) Introduction to ultrasound-guided fine-needle aspiration of the thyroid. Video Endocrinol. https://doi.org/ 10.1089/ve.2014.0003, https://www.liebertpub. com/toc/ve/1/1

9. Hen Lee Y, Hwan Baek J, Lyung SJ, Kwak JY, Jihoon KJHS, Korean Society of Thyroid Radiology, Korean Society of Radiology (2015) Ultrasoundguided fine needle aspiration of thyroid nodules: A consensus statement by the Korean society of thyroid radiology. Korean J Radiol 16(2):391-401

10. Cooper DS, Doherty GM, Haugen BR, Kloos RT, Lee SL, Mandel SJ et al (2009) Revised American Thyroid Association Management Guidelines for Patients with Thyroid Nodules and Differentiated Thyroid Cancer. The American Thyroid Association (ATA) Guidelines Taskforce on Thyroid Nodules and Differentiated Thyroid Cancer. Thyroid 19(11):1214-1167

11. Nyhsen CM, Humphreys $\mathrm{H}$, Nicolau $\mathrm{C}$, Mostbeck G, Claudon M (2016) Infection prevention and ultrasound probe decontamination practices in Europe: A survey of the European Society of Radiology. Insights Imaging 7:841-847

12. Lesourd F, Izopet J, Mervan C, Payen JL, Sandres K et al (2000) Transmissions of hepatitis $C$ virus during the ancillary procedures for assisted conception. Hum Reprod 15:1083-1085

13. Asano F, Aoe M, Ohsaki Y, Okada Y, Sasada S, Sato $S$ et al (2013) Complications associated with endobronchial ultrasound-guided transbronchial needle aspiration: A nationwide survey by the Japan Society for Respiratory Endoscopy. Respir Res 14:50

14. Lodeta B, Trkulja V (2014) Septic complications and hospital admissions after transrectal ultrasound- guided prostate biopsy: Incidence rates and outcomes in 913 consecutive biopsies. Int Urol Nephrol 46(12):2335-2336

15. M'Zali F, Bounizra C, Leroy S, Mekki Y, QuentinNoury C, Kann M (2014) Persistence of microbial contamination on transvaginal ultrasound probes despite low-level disinfection procedure. PLoS ONE 9(4):e93368

16. Koibuchi H, Kotani K, Taniguchi N (2013) Ultrasound probes as a possible vector of bacterial transmission. Med Ultrason 15(1):41-44

17. Leroy S (2013) Infectious risk of endovaginal and transrectal ultrasonography: systematic review and meta-analysis. J Hosp Infect 83(2):99-106

18. Ma ST, Yeung AC, Chan PK, Graham CA (2012) Transvaginal ultrasound probe contamination by the human papillomavirus in the emergency department. Emerg Med J30(6):472-475

19. Casalegno JS, Le Bail Carval K, Eibach D, Valdeyron ML, Lamblin G, Jacquemoud H et al (2012) High risk HPV contamination of endocavity vaginal ultrasound probes: an underestimated route of nosocomial infection? PLoS ONE 7(10):e48137

20. Mullaney PJ, Munthali $P$ et al (2007) How clean is your probe? Microbiological assessment of ultrasound transducers in routine clinical use, and cost-effective ways to reduce contamination. Clin Radiol 62(7):694-698

21. Lorentzen $T$, Nolsøe $C P$, Ewertsen $C$, Nielsen $M B$, Leen E, Havre RF, Gritzmann N, Brkljacic B, Nürnberg D, Kabaalioglu A, Strobel D, Jenssen C, Piscaglia F, Gilja OH, Sidhu PS, Dietrich CF (2015) EFSUMB Guidelines on Interventional Ultrasound (INVUS), Part I. Ultraschall Med 36:464-472

22. Müller T, Martiny $H$, Merz E, Döffert J, Wüstner $M$ Lessel W, Heynemann H, Enzmann T, Dudwiesus $\mathrm{H}$, Nuernberg D, Tesch C, Weber M, Krishnabhakdi S, Heil J, Wree A, Jenssen C (2018) DEGUM Recommendations on infection prevention in ultrasound and endoscopic ultrasound. Ultraschall in der Medizin. Europ JUltrasound 39(3):284-303

23. Bartsch DK, Luster M, Buhr HJ, Lorenz D, Germer CT, Goretzki PE (2018) Operationsindikation bei der benignen Struma des Erwachsenen. Dtsch Arztebl Int 115(1-2):1-7

24. Feldkamp J, Führer D, Luster M, Musholt TJ, Spitzweg C, SchottM (2016) Fine needle aspiration in the investigation of thyroid nodules. Dtsch Arztebl Int 113(20):353-359 